\title{
A rate-state model for aftershocks triggered by dislocation on a rectangular fault: a review and new insights
}

\author{
Rodolfo Console and Flaminia Catalli \\ Istituto Nazionale di Geofisica e Vulcanologia, Rome, Italy \\ e-mail: console@ingv.it,e-mail: catalli@ingv.it
}

November 2005

Submitted to Annals of Geophysics 


\section{Abstract}

We compute the static displacement, stress, strain and the Coulomb failure stress produced in an elastic medium by a finite size rectangular fault after its dislocation with uniform stress drop but a non uniform dislocation on the source. The time-dependent rate of triggered earthquakes is estimated by a rate-state model applied to a uniformly distributed population of faults whose equilibrium is perturbated by a stress change caused only by the first dislocation. The rate of triggered events in our simulations is exponentially proportional to the shear stress change, but the time at which the maximum rate begins to decrease is variable from fractions of hour for positive stress changes of the order of some $M P a$, up to more than a year for smaller stress changes. As a consequence, the final number of triggered events is proportional to the shear stress change. The model predicts that the total number of events triggered on a plane containing the fault is proportional to the $2 / 3$ power of the seismic moment. Indeed, the total number of aftershocks produced on the fault plane scales in magnitude, $M$, as $10^{M}$. Including the negative contribution of the stress drop inside the source, we observe that the number of events inhibited on the fault is, at long term, nearly identical to the number of those induced outside, representing a sort of conservative natural rule. Considering its behavior in time, our model does not completely match the popular Omori law; in fact it has been shown that the seismicity induced closely to the fault edges is intense but of short duration, while that expected at large distances (up to some tens times the fault dimensions) exhibits a much slower decay.

Key Words: aftershocks, earthquake triggering, fault parameters, rate-and-state, Omori law.

\section{Introduction}

Studying the space-time interaction of earthquakes is important not only for the comprehension of earthquakes, but also for its possible application to the assessment of seismic hazard.

In this paper we revisit the physical modeling of the interaction between seismic events, seeking a relationship between the source parameters of an earthquake and the rate of all other earthquakes that follow it. The results obtained from the simulations performed with 
the physical model proposed in this study are not totally innovative; however, they are rarely taken into account by the numerous studies concerning the fault interaction and earthquake triggering.

A purely statistical approach to this phenomenon exists with the name of "epidemic model" (Ogata, 1988, 1998; Console et al., 2003). In the epidemic model each earthquake source is supposed capable of increasing the probability of new earthquakes according to a spatiotemporal kernel representing the probability distribution, which is decreasing from the causative source. The epidemic model is applicable not only to the aftershock phenomenon but also to the foreshock. However, despite it provides an accurate statistical representation of earthquake interaction, the physical interpretation is neglected.

In order to formulate a more physical description of clustered seismicity, we consider that seismic events modify the stress field around the causative fault. Our main goal in this work is to observe the spatio-temporal variation of the seismicity rate after an event introducing in the algorithm a rate-state model approach derived by Ruina (1983) and Dieterich (1986, 1992, 1994). In particular, we seek a physically based relationship between the magnitude of the causative earthquake and the number of its aftershocks. Some recent studies put in evidence that sudden stress variations, even of small magnitude, can produce large variations of the seismicity rate. It is recognized as a phenomenon of triggering on faults that are already loaded by tectonic stress and in close-to-failure condition. The seismicity rate increases in general where the stress change (called Coulomb Stress Change) is positive, according to the Coulomb model (Mendoza and Hartzell, 1988; Boatwright and Cocco, 1996; Stein et al., 1997; Gomberg et al., 1998, 2000; Harris, 1998; King and Cocco, 2001; Stein, 1999; Toda and Stein, 2000; Kilb et al., 2002; Belardinelli et al., 2003; Nostro et al., 2005). These studies were able to give a physical interpretation for earthquake interaction observed in specific real cases, but were not suitable for the general application of the model in predictive way that can be tested for example using the information contained in a seismic catalog. In particular, the Coulomb stress change criterion does not allow to model the hyperbolic time decay of the aftershock rate after a mainshock, known as the Omori Law. In this simple model all the impending earthquakes would be clock-advanced by a constant time that depends on the stress change and on the loading 
rate but does not depend on the physical properties of the fault (Gomberg et al., 1998). The experience shows that the Earth does not rather behave in this way. The most popular empirical description of the phenomenon is the Omori law, stating that the aftershock rate decays in time as $t^{-1}$. Among the various theories modeling the Omori law, we have taken in consideration only the one that assumes the rate-and-state dependent constitutive model of faults. We have considered only the static stress changes created by an earthquake. We have not considered, even without rejecting them in principle, other hypotheses that predict a time variation in stress, like viscoelastic relaxation or the diffusion of fluids in the crust. The rate-state model for earthquake nucleation seems capable of substantially explaining all the phenomenology. In this model a promoted failure does not occur instantaneously at the exceeding of a threshold stress as for the Coulomb-Amonton criterion, but follows a more complex non-linear time history with different phases. This time history depends on the physical properties of the fault too. Gomberg et al. (2005) have shown that the rate-state Dieterich model may be derived from simple general ideas. The application of the rate-and-state model to the evolution of seismicity is not new; Toda et al. (1998) have used this model to predict the spatio-temporal seismicity rate after the Kobe earthquake. Other examples of application of the same model can also be found in the literature (Toda and Stein, 2000, 2002, 2003). Following the rate-state model it has recently been possible to simulate seismicity quite realistically, accounting for the rate of events triggered by stress changes even at large distance from the inducing earthquake (Ziv, 2003; Ziv and Rubin, 2003).

Basing our work on the classical theory of the elasticity, we start from the formulation of the stress released by a rectangular fault with uniform stress-drop; then we apply the rate-state model (Dieterich, 1994) obtaining the complete time and space distribution of the seismicity induced by a given earthquake. It is important to remark that for present purposes we neglect the interaction between subsequent events, supposing that only the first event perturbs the stress field. We also neglect the effect of the free surface of the Earth. In this study we focus our attention on the behavior of induced seismicity in space (on the fault plane) and time and its relationship with the fault's parameters. The results of simulations are analyzed to find out the scaling relationships existing between the free parameters of the model and the expected 
seismicity in a way that will allow the validation of the model by real observations.

\section{Elastic model for a rectangular fault}

The theoretical approach of this work has been made as simple as possible, still preserving the capability of modeling the stress transfer from a seismic source to another.

Suppose that a fault is embedded in a homogeneous, isotropic, elastic medium where the stress tensor $\sigma$ is uniform. At a particular moment, the fault slips generating an earthquake, and suppose that the earthquake fully releases the component of the traction parallel to the slip vector across the fault. We call $\Delta \sigma$ (the stress drop) the uniform negative change of traction on the fault. It is well known that the slip distribution on a fault in similar conditions is not uniform. The slip distribution will satisfy the theory of the elasticity at the new equilibrium, and it will be zero on the edges of the fault. It is also well known that the shear stress on the fault plane outside the edges of the fault increases significantly.

The analytical solution for the slip distribution on a fault at the equilibrium does not exist except that for very simple geometries, such as a rectangular fault of constant width and infinite length (Knopoff, 1957) and for a circular fault (Keilis-Borok, 1959; Udias, 1999). As mentioned by Kostrov and Das (1998), the analytical solution is not known even for a geometry as simple as a rectangular shape of width $W$ and length $L$. In the literature it is possible to find the computation of the stress field generated by an ideal rectangular fault with uniform slip in an infinite homogeneous space or half-space (Chinnery, 1961; Iwasaki and Sato, 1979; Okada, 1985, 1992). However, as mentioned above, this is not a realistic model since it implies the com-penetrability of the medium at the edges of the slip region. For this reason, here we make use of a non uniform slip distribution that is approximatively compatible with a uniform stress drop on the fault.

To calculate the static displacement, stress and strain, we start from the Somigliana Tensor and we make use of the double couple point source elastic theory. We compute the modified Coulomb failure stress from the relation:

$$
\sigma_{c}=T_{s}+\mu^{\prime} T_{n}
$$


where $T_{s}$ and $T_{n}$ are respectively the traction parallel to the slip vector and the traction normal to the fault plane (negative for compression); $\mu^{\prime}$ is the effective coefficient of friction incorporating the pore fluid pression that is commonly assumed to be proportional to normal stress change (although this assumption is not general, see Cocco and Rice (2002)). The effective coefficient of friction in coseismic stress change studies has varied from 0.0 to 0.75 , with an average value of $\mu^{\prime}=0.4$ often used. This is the value we have used in our simulations.

Our model of an extended source is drawn heuristically from that of a rectangular fault infinitely extending in one direction and that of a circular fault, and it is characterized by a slip distribution as:

$$
\Delta u=\frac{\Delta \sigma}{\mu} \sqrt{\frac{\left[\left(\frac{W}{2}\right)^{2}-x^{2}\right]\left[\left(\frac{L}{2}\right)^{2}-y^{2}\right]}{\frac{L W}{4}}},
$$

defined for $-W / 2 \leq x \leq W / 2$ e $-L / 2 \leq y \leq L / 2$, where $x, y$ are the coordinates on the fault plane whose origin is coincident with the center of the rectangle, $\Delta \sigma$ is the stress drop and $\mu$ the shear modulus (rigidity) of the elastic medium. Equation (2) satisfies the condition of zero slip on the edges of the rectangle. The slip distribution is of a form very similar to that obtained numerically by Kostrov and Das (1998) with the finite differences method. Its analytical form is similar to the solution for a circular fault. Moreover, for a fault of square shape $(W=L)$ it achieves a seismic moment consistent with those obtained analytically for two circular faults, respectively inscribed and circumscribed to the square. Bonafede and Neri (2000) have shown that, when imposing a unidirectional traction release in the strike or dip direction, a minor component of slip is present, over the fault plane, even in the direction perpendicular to the released traction. We have neglected this component.

For our numerical applications we discretize the continuous slip distribution of equation (2) with a set of point sources densely and uniformly distributed on the fault. The more numerous are the point sources, the more accurate will be the approximation.

In our numerical simulations, we arbitrarily, but without loss of generality, suppose that the fault is on the $\left(x_{1}, x_{3}\right)$ plane. Let us notice that some fault parameters are connected among each other; in particular, the scalar moment $M_{0}$ is defined through the relation:

$$
M_{0}=\mu \overline{\Delta u} W L=\frac{\pi^{2}}{16} \mu \Delta u_{\max } W L=\frac{\pi^{2}}{32} \Delta \sigma(W L)^{3 / 2}
$$


where $\overline{\Delta u}$ is the average slip on the fault and the maximum slip, $\Delta u_{\max }$, is defined as:

$$
\Delta u_{\max }=\frac{\Delta \sigma}{\mu} \frac{\sqrt{L W}}{2} .
$$

We also assume that the magnitude $M$ is proportional to the logarithm of the seismic moment $M_{0}$ (measured in $\mathrm{Nm}$ ) according to the Kanamori and Anderson (1975) relation:

$$
\log M_{0}=9.1+1.5 M
$$

The computer code allows the user to chose among several options: - The focal mechanism characterizing the causative fault and the induced events constrained by the strike $\phi$, the $\operatorname{dip} \delta$ and the rake $\lambda$ angles; - Dimensions and stress drop, seismic moment, or magnitude of the causative fault; - Type of output: displacement (3 components), stress or strain (6 independent components), traction parallel to the slip vector and perpendicular to the fault plane (2 component) and Coulomb failure stress (1 component).

The output values are given on a rectangular grid of points, whose orientation (horizontal, or vertical parallel to one of the co-ordinate axes), dimensions, and spacing are also selected by the user. See Figure 1 for the conventions we adopted for the reference system and the angles.

In this work we make, for sake of simplicity, the assumption that all the aftershocks have the same focal mechanism of the causative earthquake.

Figure 2 shows the component of the stress field $\sigma_{12}$ parallel to the slip calculated through the parameters listed in Table 1. Note that this figure shows the stress change and not its absolute value.

Figure 2 clearly shows that the shear stress $\sigma_{12}$ is maximum near the edges of the fault, in particular along the slip direction $x_{1}$, and decreases with increasing the distance from the fault. Inside the fault the stress change is negative and approximately equal to the imposed value of $-\Delta \sigma$.

\section{The rate-state model}

As already stated, we assume that the time-dependent behavior of the seismicity triggered by a shear stress change in a population of faults is described by the rate-state model introduced by 
Dieterich $(1992,1994)$ where the constitutive model proposed by Ruina (1983) and Dieterich (1986) is used. According to this model the rate $R$ of earthquakes due to a $\Delta \tau$ shear stress perturbation is given by:

$$
R=\frac{r \frac{\dot{\tau}}{\dot{\tau}_{r}}}{\left[\frac{\dot{\tau}}{\dot{\tau}_{r}} \exp \left(\frac{-\Delta \tau}{A \sigma}\right)-1\right] \exp \left(\frac{-t}{t_{a}}\right)+1} \quad \dot{\tau} \neq 0
$$

whose various parameters are defined in Table 2. Note that equation (6) does not account for the magnitude of the earthquakes, for the distribution of which we assume the classical GutenbergRichter frequency-magnitude relationship. However, both $R$ and $r$ refer to the number of earthquakes in a specific magnitude range.

We make the assumption $\dot{\tau}=\dot{\tau_{r}}$. The meaning of this assumption is that the primary earthquake does not change the long-term stress rate due to the tectonic driving forces in the environment.

It is easily understandable from (6) that the integral of $R$ over infinite time diverges. This is due to the fact that the limit of $R$ for $t \rightarrow \infty$ is the background rate $r$. We prefer to deal with the net triggered seismicity rate $R^{\prime}=R-r$. The plot of $R^{\prime}$ versus time for different values of $\Delta \tau$ is shown in Figure 3 (see also Dieterich (1994)). It shows that the initial value of the triggered seismicity rate, immediately after the triggering event, is proportional to the exponential of the stress variation but the time period over which $R(t)$ is maximum and relatively constant decreases exponentially by the same factor for increasing shear stress change. This time period, in substantial agreement with Dieterich (1994), can be obtained computing the intersection between $R^{\prime}(t=0)$ and the straight line corresponding to the limit of $R^{\prime}$ when $\Delta \tau \rightarrow \infty$. The difference with Dieterich (1994) is that we have preferred to make use of $R^{\prime}$, rather than $R$, and we ignore the case $\dot{\tau}=0$ which we judge not realistic. Such time period ranges from few hours for a variation $\Delta \tau=5 M P a$ to nearly one year for $\Delta \tau=1 M P a$. Figure 3 finally shows that the total duration of the triggered seismicity is the same but the shape of the decay is different for different $\Delta \tau$.

The integration of $R^{\prime}$ over infinite time leads to the not completely trivial result that the total number of triggered events in an area of constant stress change is proportional to $\Delta \tau$ : 


$$
\int_{0}^{\infty} R^{\prime}(t) d t=\frac{r t_{a}}{A \sigma} \Delta \tau=\frac{r}{\dot{\tau}} \Delta \tau
$$

This relationship, predicted by the friction model, had been observed for "Landers Aftershocks" by Gross and Kisslinger (1997).

Consider the integral over time of the triggered seismicity rate $R^{\prime}$, once for a positive $\Delta \tau$, and then for a negative $\Delta \tau$ of the same size, and we sum the two functions. We obtain a quantity that initially has a positive increase and then tends back to zero (Figure 4). This result can be interpreted as a sort of conservative law of the seismicity, by saying that the expected total number of aftershocks generated in a place close to a fault can be balanced, at long range, by the total number of those events that are inhibited by the stress drop in a place internal to the fault if the stress drops are equal in absolute value. It seems to us that this circumstance has been ignored, so far, in all previous studies on this subject.

For numerical applications it is necessary to define the value of the various parameters appearing in equation (6). Table 3 contains a list of values inferred from several geophysical considerations and used in our simulations. The reference seismicity rate, $r$, is a quantity that should be known experimentally for a given area, and it is related to the average strain rate $\dot{\tau}$ of that area. We use for $r$ a uniform value of four events of magnitude exceeding 3.0 per year per $1000 \mathrm{~km}^{2}$, and for $\dot{\tau}$ a value of $5 \mathrm{KPa}$ per year. These are simplifications of the reality where $r$ and $\dot{\tau}$ are not geographically uniform. These parameters seem however reasonable for an area of moderate seismicity. The reason why $r$ is expressed as a number of events per unit area and time is given below. The parameter $A$ of the constitutive law has a value ranging from 0.005 to 0.015 obtained from laboratory experiments (Dieterich, 1995). Generally, simulations that most nearly resemble earthquakes in nature were obtained with rather small values of $A$, near to $A \approx 0.001$. The value of $A \sigma$ has been evaluated for different sequences of earthquakes by several authors (Stein, 1999; Belardinelli et al., 2003). The parameter $\sigma$ is the normal stress on the fault (of the order of some tens of $M P a$ ). The characteristic time of aftershocks, $t_{a}$, depending on the other parameters through the relation $t_{a}=(A \sigma) / \dot{\tau}$, in this context is of the order of several tens of years. This value is essential to model a long term triggered seismicity, because the effect of the stress change disappears completely after a time of the order of the 
double of $t_{a}$.

\section{Application to a rectangular fault}

Dieterich (1994) discussed in detail the case of a finite circular dislocation with uniform stress drop. In our simulations we have taken into consideration a vertical, rectangular strike-slip fault embedded in an infinite, homogeneous, isotropic, elastic medium. The values assumed for the fault parameters are again those reported in Table 1. Assuming a constant stress drop $\Delta \sigma$, the scalar seismic moment $M_{0}$ is proportional to the quantity $(W L)^{3 / 2}$, that can be considered a sort of equivalent source volume. Scaling $W$ and $L$ by a given factor, $M_{0}$ scales accordingly, and also the shape of the stress distribution in space scales by the same factor. Assuming that the background seismicity rate is given as the number of events per unit volume and time, and integrating over the space, we find that the number of events triggered by the earthquake is proportional to the source volume, i.e. to the seismic moment $M_{0}$, or to $10^{\frac{3}{2} M}$, where $M$ is the magnitude. In this study we analyze only those aftershocks occurring on the fault plane neglecting off-fault seismicity and assuming that this is the plane of weakness optimally oriented with respect to the tectonic loading stress. The modeling of the space-time distribution of seismicity triggered by an earthquake is straightforward, introducing the expression for the Coulomb failure stress $\sigma_{c}$ in equation (6) for $\Delta \tau$ considering that on the fault plane $\sigma_{c} \equiv \Delta \tau$. In fact, on the fault plane, the normal stress changes in a homogeneous and isotropic medium are likely to vanish.

In this case, the number of triggered events would be proportional to the fault area of the triggering event and then to $M_{0}^{2 / 3}$, or to $10^{M}$. If this scaling is true, one earthquake of magnitude $M$ would have a number of aftershocks ten times larger than one earthquake of one magnitude unit smaller. Assuming, then, that the $b$ value of the Gutenberg-Richter frequencymagnitude relation is equal to 1 , it would follow that the earthquakes of magnitude $M$, all together, produce the same number of aftershocks as the earthquakes of one magnitude unit smaller. This hypothesis is supported by the statistical analysis of real observations (Utsu, 1969; Yamanaka and Shimazaki, 1990; Felzer et al., 2001; Helmstetter, 2003; Helmstetter et al., 2005) and will be used in the following of the paper. 
A first group of simulations concerning the seismicity triggered by a fault as a whole have been performed to study the variation of the total number of triggered events (in space and time), $N_{\text {tot }}$, versus the main free source parameters: the stress drop $\Delta \sigma$, the linear dimensions $L$ and $W$ and the magnitude $M$.

Tables 4, 5 and 6 show the results obtained assigning a fixed value for one of the parameters at a time, and letting the others change according to equations (3) and (4). These simulations have been carried out by summation of the single contributions of the elementary cells constituting the area surrounding the fault. Only the seismicity triggered on the fault plane, but outside the fault edges, has been considered. Looking at Table 4 one can notice that, under our hypotheses, for a constant stress drop, the total number of triggered events increases about proportionally to the fault area $L W$. Theoretically it should be exactly proportional to the area of the source, but in the calculations there are numerical imprecisions due to the grid size approximations. These numerical simulations show, also, that the number of aftershocks is proportional to the $2 / 3$ power of the scalar seismic moment or to the exponential of the magnitude $\left(10^{M}\right)$, in agreement with the above theoretical arguments. Table 5 clearly shows the proportionality existing between the stress drop $\Delta \sigma$ and $N_{t o t}$, having fixed the linear dimensions of the fault. This is a simple consequence of the proportionality between the stress drop, $\Delta \sigma$, and the shear stress change, $\Delta \tau$, at any point of the space around the source; furthermore, it is an example of the proportionality expressed in equation 7 between $\Delta \tau$ and the total number of triggered events, integrated on the whole space. The results reported in Table 6 give credit to the hypothesis that, for a constant magnitude, the earthquakes of smaller linear dimensions generate a larger number of triggered events. Indeed, a point source would yield the largest quantity of triggered events for the same magnitude.

Let us mention, lastly, the results of a simple numerical test. We just have theoretically shown in the previous section that the total number of events triggered by a stress change $\Delta \tau$ per unit area is, after a time much larger than the characteristic time $t_{a}$, identical to the number of events inhibited by a negative shear stress change $-\Delta \tau$ on the same area. The numerical simulations about an extended source, with parameter still shown in Table 1, reflect this idea in a more general way. In fact, if we take the total number of aftershocks produced in a time 
interval of 100 years by a fault of $100 \mathrm{~km}^{2}$ on the portion of an area of $10^{4} \mathrm{~km}^{2}$ external to it, we find 353.2 events, while the same computation made including the negative contribution of the area internal to fault itself gives only 15.6 (less than 5\%). The idea of an overall null balance is, then, substantially met: the phenomenon of clustering does not change the long term seismicity rate of a large area. This is in contrast with the opinion that trigger zones produce many more shocks than are missing from the shadows (Toda and Stein, 2003). We nevertheless regard for Marsan (2003) that put in evidence the practical difficulty to observe in nature the lack of events (the quiescence) mainly when looking at weakly active regions and short timescales.

\section{Temporal behavior of the triggered seismicity}

Let us now consider in more detail the dependence on time of the number of triggered events predicted by the present hypothesis. Kagan and Jackson (1991) and Dieterich (1994) found, in their empirical works, that shallow aftershocks, above a given magnitude cutoff, decay globally (for different distance intervals) in about 10 years and this time decay appears to systematically decrease with increasing depth. In our model the behavior in different zones depends on the different values of the stress changes, that decrease with the increasing distance from the edges of the fault. It can be then noted that the plateau of the plot of the seismicity rate versus time (see Figure 3) has a duration that increases with the increasing distance from the source. This result depends on our assumption of considering only aftershocks on the mainshock fault plane. It can also be biased by the fact that we consider only the effect of the static stress step $\Delta \tau$ caused by a mainshock and we neglect the interaction between others events. We also do not account for heterogeneities of frictional or constitutive properties nor for the dynamic stress concentration at the crack tip. In spite of that, we believe that our model can describe a behavior not so far from reality. Therefore we keep as our reference the model of fault given in Table 1 again.

As shown in Figure 5a, in the close neighboring of the fault (within $0.5 \mathrm{~km}$ from the perimeter of the fault), most of the aftershocks occur within one day after the occurrence of the inducing earthquake, and more than $1 / 3$ of them occur already in the first hour. This is clearly 
not the case for further distance from the source: in the slice between 0.5 and $8.5 \mathrm{~km}$ from the fault, about half of the activity is exploited within about one year. The further we go apart from the source, the longer is the time interval necessary for the exploitment of the triggered activity. In fact, in the distant zone (from 20 to $100 \mathrm{~km}$ from the fault) most of the triggered events occur longer than 20 years after the inducing earthquake (Figure 5b). The behavior shown in Figure 5a and 5b is illustrated also by the snapshots of the spatial distribution of the seismicity rate at different times. Figures 6,7 and 8 clearly show how the distribution of aftershocks resembles that of the stress change (Figure 2) and how the time decay is different in the different slices. These also show how shadow zones (where the seismicity is inhibited) have an effect longer in time. It should be noted again that we make the assumption that the aftershocks occur mainly on the fault plane and that these have the same focal mechanism of the causative event.

We are now interested to check if the generalized Omori law is suitable to describe the temporal behavior of $R^{\prime}(t)$ predicted by our model in every spatial slice and, in such case, by means of which free parameters. We refer to the generalized Omori law as to the formula describing the decay of aftershock rate after a mainshock (Utsu et al., 1995) through three free parameters $a, c$ e $p$ :

$$
R^{\prime}(t)=\frac{a}{(c+t)^{p}}
$$

For this purpose we make use of an algorithm for the least squares best-fit of the sets of data reported in Figure 5 by the integral of (8) over the time:

$$
y=\int_{0}^{t} R^{\prime}\left(t^{\prime}\right) d t^{\prime}=a\left[\frac{(c+t)^{1-p}-c^{1-p}}{1-p}\right] .
$$

The results obtained for the best-fit are shown in Table 7 . It is evident that the values of the parameters obtained are quite different from case to case (for instance, the $c$ parameter is extremely small for the slices closest to the fault edges), and that the standard deviation is larger for the slices that include the wider range of distances from the fault. These results show that the same set of parameters in equation (9) can not fit the synthetic values obtained for different zones. Dieterich (1994) reached a similar conclusion for a source model represented by a circular shear crack. However, his analysis was limited to the spatial variation of $p$ parameter letting $c=0$. The observation that the Omori $p$ parameter varies spatially in real aftershocks 
sequences has been documented not only by Dieterich (1994) but also in a number of papers (Wiemer and Katsumata, 1999; Wiemer, 2000; Enescu and Ito, 2002; Wiemer et al., 2002; Wiemer and Wyss, 2002). Wiemer et al. (2002) reported a case of significantly different $c$ values in the northern and southern Hector Mine aftershock volume, respectively. Enescu and Ito (2002) in their study of aftershock activity of the 2000 Western Tottory earthquake found c-values very close to 0 except for larger magnitude earthquakes, arguing that this feature could result from incompleteness of data, or might also reflect the complexity of the rupture process.

The situation is illustrated also by the plots of Figures 9 and 10, showing the graphical comparison between the synthetic data and the relative theoretical approximation for different slices.

\section{Conclusions}

In the development of this model some approximations have been made. Two of the most important are (a) having neglected the dishomogeneity of the stress drop on the fault, and consequently the very complicated pattern of slip (this must certainly have influence on the aftershock pattern: some aftershocks may even occur inside the fault, where the gradient of slip is high); (b) having neglected the interaction between subsequent events.

The first step of our modeling, concerning the stress change in the medium following an earthquake, has given results comparable to those of previous papers as, for instance, Chinnery (1961) and King and Cocco (2001). This substantial agreement, though the simplifications introduced in this specific case of rectangular source, supports the validity of our methodology.

Neglecting the interaction among seismic events and using the rate-and-state model of Dieterich (1994) entails that the total number of triggered events per unit area is proportional to the shear stress change. Nevertheless, the time constant by which the rate of events decreases has a large variability, ranging from a fraction of hour for a shear stress change of several $M P a$, up to tens of years for stress variations smaller than $1 \mathrm{MPa}$.

A first set of simulations carried out with a model of rectangular fault, based on the hypothesis that most of the aftershocks occur on the same plane of the fault, has lead to the relationship between the main source parameters and the number of triggered events. The 
simulations lead to the conclusion that many earthquakes of small magnitude can produce, in total, a number of aftershocks comparable to that of fewer larger earthquakes. Our model predicts, in fact, that, for a constant stress drop, the number of triggered events is roughly proportional to the seismic moment at the power of $2 / 3$, or equivalently to $10^{M}$, as observed in various occasions. Helmstetter (2003); Helmstetter et al. (2005) suggest that this behavior can be related to the fractal structure of the spatial distribution of the seismicity and to the nature of earthquake interactions. For a costant stress drop we also observe that the total number of triggered events is proportional to the area of the fault; on the other way, if the linear dimensions of the fault are kept constant, the number of triggered events is proportional to the stress drop.

An important aspect of the model concerns the time-space behavior of the triggered seismicity. The simulations show that the application of the rate-state model and equation (6) introduced by Dieterich (1994) to the static model of stress change has as a consequence something more complicate than the conventional Omori law for the temporal decay of aftershock rate. In fact, the seismicity is expected to be intense (in accordance to the stress change) and have a maximum constant value, close to the fault edges of the primary earthquake, but the time necessary for the starting of decay is shorter. On the contrary, the seismicity expected at larger distances (up to 10 times the linear dimensions of the fault) is weak, but the time necessary for the decay from the maximum value is longer (even some tens of years). We can conclude that the temporal behavior of aftershock rate varies in space. Moreover, the model predicts that the total long term production of aftershocks, including wider areas, is not negligible respect to the short term production. In this respect, the regional background seismicity could be interpreted as a sort of noise, or memory, due to the superposition of the effect of many older earthquakes. The time decay described by the popular Omori law could be interpreted as an apparent average result of the contribution from the various areas on the plane containing the primary fault.

The rate-state model (Dieterich, 1994) used in our algorithm for the time distribution of the seismicity produced by a dislocation on a rectangular fault, has allowed a partial justification of the Omori law. We outline the following main conclusions obtained from the simulations: 
- The seismic activity is very intense during the first few hours or days after the occurrence time of the primary earthquake, in the rectangular slice close to the edges of the fault;

- The immediate decay of this intense activity is followed by a period of time during which the activity at larger distance (of the order of the linear dimensions of the fault) is nearly constant. For longer time (several years) the total number of triggered events in the external zone becomes comparable with that of the most internal one.

- the number of events triggered over a long time scale at distances larger than the fault linear dimensions is significantly large;

- the seismic activity inside the fault drops to negligible values at the occurrence of the primary earthquake and returns to normal values only after a time much longer than the characteristic time (several tens of years in our simulations); for a long time range the number of events inhibited inside the fault is comparable to that of the events triggered outside: the net balance can be considered null; the seismicity rate changes in space and time, but it does not have influence on the rate averaged over very large intervals of space and time.

In light of the consequences derived from our model, both positive and negative perturbations of the seismic activities resulting from the interactions among earthquakes would last for a time of the order of decades. In this respect, it seems unrealistic to define and observe what is commonly called "background seismicity" of an area.

We can conclude that the model here analyzed, in spite of its very simple conception, gives a physical justification both to the very popular phenomenon of short term earthquake clustering (aftershocks and foreshocks in strict sense) and to that of the long term induction and quiescence, also observed in various occasions.

We are confident that the refinement of the model and its validations with real catalogs will bring to even more interesting consequences. 


\section{Acknowledgments}

We are grateful to Massimo Cocco and Maria Elina Belardinelli for the stimulating discussions and their criticism which led to a significantly better version of the paper. We thank Tom Parsons for many useful suggestions to help make this paper clear. 


\section{Authors}

R. Console,

Istituto Nazionale di Geofisica e Vulcanologia,

via di Vigna Murata, 605

00142, Rome, Italy

mailto: console@ingv.it

phone:+3906 51860417

fax:+3906 5041181

\section{F. Catalli,}

Istituto Nazionale di Geofisica e Vulcanologia,

via di Vigna Murata, 605

00142, Rome, Italy

mailto: catalli@ingv.it

phone:+390651860571 


\section{Tables}

Table 1: Source parameters used in numerical applications.

\begin{tabular}{ll}
\hline Parameter & Value \\
\hline Strike, dip and rake & $0,90,0$ \\
Dimension of the fault & $10 \mathrm{~km} \times 6 \mathrm{~km}$ \\
Spacing on the fault & $0.1 \mathrm{~km}$ \\
Dimension of the grid & $30 \mathrm{~km} \times 30 \mathrm{~km}$ \\
Spacing on the grid & $0.5 \mathrm{~km}$ \\
Magnitude & 6 \\
\hline
\end{tabular}

Table 2: Constitutive parameters used in Dieterich's relation.

\begin{tabular}{ll}
\hline Symbol & Description \\
\hline$r$ & reference seismicity rate \\
$\dot{\tau}$ & shear stressing rate \\
$\dot{\tau}_{r}$ & reference stressing rate \\
$\Delta \tau$ & earthquake shear stress change \\
$A$ & fault constitutive parameter \\
$\sigma$ & normal stress \\
$t$ & time \\
$t_{a}$ & characteristic time for seismicity to return to the steady state \\
\hline
\end{tabular}


Table 3: Values of the parameters used in the numerical applications of the model for induced seismicity.

\begin{tabular}{ll}
\hline Parameter & Value \\
\hline$r$ & 4 events $/\left(y \cdot 1000 \mathrm{~km}^{2}\right)$ \\
$\dot{\tau}$ & $5 \mathrm{KPa}$ \\
$\dot{\tau}_{r}$ & $5 \mathrm{KPa}$ \\
$A$ & 0.008 \\
$\sigma$ & $30 \mathrm{MPa}$ \\
$t_{a}$ & $48 \mathrm{y}$ \\
\hline
\end{tabular}


Table 4: Relation between fault parameters and aftershock production. The value of $\Delta \sigma$ is fixed at $4 \mathrm{MPa}$; the grid used in this computation has an area of $10^{4} \mathrm{~km}^{2}$, the spacing on it is $0.5 \mathrm{~km}$. The spacing on the fault is $0.05 \mathrm{~km}$.

\begin{tabular}{|c|c|c|c|}
\hline$L \cdot W\left[\mathrm{~km}^{2}\right]$ & $M$ & $M_{0}[\mathrm{Nm}]$ & $N_{\text {tot }}$ \\
\hline 4 & 4.6 & $9.9 \cdot 10^{15}$ & 13.4 \\
25 & 5.4 & $1.5 \cdot 10^{17}$ & 78.2 \\
100 & 6.0 & $1.2 \cdot 10^{18}$ & 263.2 \\
225 & 6.4 & $4.2 \cdot 10^{18}$ & 532.8 \\
\hline
\end{tabular}

Table 5: Relation between fault parameters and aftershock production. The area of the fault is fixed at the value of $100 \mathrm{~km}^{2}$, the spacing is of $0.05 \mathrm{~km}$; the grid used in the computation has an area of $17 \times 17 \mathrm{~km}^{2}$, the spacing on it is of $0.5 \mathrm{~km}$.

\begin{tabular}{|c|c|c|c|}
\hline$\Delta \sigma[M P a]$ & $M$ & $M_{0}[N m]$ & $N_{\text {tot }}$ \\
\hline 1 & 5.6 & $3.1 \cdot 10^{17}$ & 51.8 \\
2 & 5.4 & $6.2 \cdot 10^{17}$ & 103.4 \\
3 & 5.9 & $9.3 \cdot 10^{17}$ & 155.2 \\
4 & 6.0 & $1.2 \cdot 10^{18}$ & 206.8 \\
5 & 6.1 & $1.6 \cdot 10^{18}$ & 258.6 \\
\hline
\end{tabular}

Table 6: Relation between fault parameters and aftershock production. The value of $M$ is fixed at 6.0; the output grid is of $20 \mathrm{~km} \times 20 \mathrm{~km}$, the spacing is $0.5 \mathrm{~km}$. The spacing on the fault is $0.05 \mathrm{~km}$.

\begin{tabular}{|c|c|c|}
\hline$L \cdot W\left[\mathrm{~km}^{2}\right]$ & $\Delta \sigma[M P a]$ & $N_{\text {tot }}$ \\
\hline 4 & 510.7 & overflow \\
25 & 32.7 & 597.6 \\
100 & 4.1 & 223.2 \\
225 & 1.2 & 107.8 \\
\hline
\end{tabular}


Table 7: Results obtained for the best-fit of the sets of data reported in Figure 5 by the relation (9). The symbol rms indicates the standard deviation.

\begin{tabular}{c|cccc} 
Slice & $a\left[y^{p-1}\right]$ & $c[y]$ & $p$ & $r m s$ \\
\hline$(0 \div 0.5) \mathrm{km}$ & $3.24 \pm 0.17$ & $0.37 \cdot 10^{-7} \pm 0.2 \cdot 10^{-6}$ & $1.02 \pm 0.02$ & 1.86 \\
$(0 \div 8.5) \mathrm{km}$ & $11.0 \pm 0.76$ & $0.23 \cdot 10^{-7} \pm 0.2 \cdot 10^{-6}$ & $0.91 \pm 0.032$ & 9.16 \\
$(0.5 \div 8.5) \mathrm{km}$ & $83.7 \pm 70.0$ & $3.1 \pm 1.96$ & $1.44 \pm 0.19$ & 3.3 \\
$(0 \div 20) \mathrm{km}$ & $34.3 \pm 8.0$ & $0.13 \cdot 10^{-1} \pm 0.019$ & $1.14 \pm 0.077$ & 9.22 \\
$(0 \div 100) \mathrm{km}$ & $36.8 \pm 9.1$ & $0.15 \cdot 10^{-1} \pm 0.023$ & $1.12 \pm 0.08$ & 10.8 \\
$(20 \div 100) \mathrm{km}$ & $12.5 \cdot 10^{3} \pm 10.9 \cdot 10^{3}$ & $61.0 \pm 5.43$ & $2.8 \pm 0.96 \cdot 10^{-3}$ & 0.45
\end{tabular}




\section{References}

Belardinelli, M., A. Bizzarri, and M. Cocco (2003), Earthquake triggering by static and dynamic stress change, J. Geophys. Res., 108(B3), 2135, doi:10.1029/2002JB001779.

Boatwright, J., and M. Cocco (1996), Frictional constraints on crustal faulting, J. Geophys. Res., $101(10)$, 13,895-13,910.

Bonafede, M., and A. Neri (2000), Effects induced by an earthquake on its fault plane: a boundary element study, Geophys. J. Int., 141(1), 43-56, doi:10.1046/j.1365-246X.2000.00074.x.

Chinnery, M. (1961), The deformation of the ground around surface faults, Bull. Seism. Soc. Am., 51, 355-372, doi:10.1046/j.1365-246X.2000.00074.x.

Cocco, M., and J. R. Rice (2002), Pore pressure and poroelasticity effects in coulomb stress analysis of earthquake interactions, J. Geophys. Res., 107(B2), 2030, doi:10.1029/2000JB000138.

Console, R., M. Murru, and A. Lombardi (2003), Refining earthquake clustering models, J. Geophys. Res., 108(B10), 2468, doi:10.1029/2002JB002130.

Dieterich, J. (1986), A model for the nucleation of earthquake slip, Geophy. Monogr. Ser., 37, $36-49$.

Dieterich, J. (1992), Earthquake nucleation on faults with rate and state dependent strenght, Tectonophysics, 211, 115-134.

Dieterich, J. (1994), A constitutive law for rate of earthquake production and its application to earthquake clustering, J. Geophys. Res., 99(18), 2601-2618.

Dieterich, J. (1995), Earthquake simulations with time-dependent nucleation and long-range interactions, Nonlinear Processes in Geophysics, 2, 109-120.

Enescu, B., and K. Ito (2002), Spatial analysis of the frequency-magnitude distribution and decay rate of aftershock activity of the 2000 Western Tottori earthquake, Earth Planet Space, $54,847-859$. 
Felzer, K. R., T. W. Becker, R. E. Abercrombie, G. Ekstrm, and J. R. Rice (2001), Triggering of the 1999 MW 7.1 Hector Mine earthquake by aftershocks of the 1992 MW 7.3 Landers earthquake, J. Geophys. Res., 10(B9), 2190, doi:10.1029/2001JB000911.

Gomberg, J., N. Beeler, M. Blanpied, and P. Bodin (1998), Earthquake triggering by transient and static deformations, J. Geophys. Res., 103(12), 24,411-24,426.

Gomberg, J., N. Beeler, and M. Blanpied (2000), On rate-state and Coulomb failure models, J. Geophys. Res., 105(14), 7857-7872.

Gomberg, J., P. Reasenberg, M. Cocco, and M. Belardinelli (2005), A frictional population model of seismicity rate change, J. Geophys. Res., 110(B05S03), doi:10.1029/2004JB003404.

Gross, S., and C. Kisslinger (1997), Estimating tectonic stress rate and state with Landers aftershocks, J. Geophys. Res., 102(B4), 7603-7612.

Harris, R. (1998), Introduction to special section: Stress triggers, stress shadows, and implications for seismic hazard, J. Geophys. Res., 103(12), 24,347-24,358.

Helmstetter, A. (2003), Is earthquake triggering driven by small earthquakes?, Phys. Res. Lett., $91(058501)$.

Helmstetter, A., Y. Kagan, and D. Jackson (2005), Importance of small earthquakes for stress transfers and earthquakes triggering, J. Geophys. Res., 110(B05S08), doi: 10.1029/2004JB003286.

Iwasaki, T., and R. Sato (1979), Strain field in a semi-infinite medium due to an inclined rectangular fault, J. Phys. Earth, 27, 117-123.

Kagan, Y. Y., and D. D. Jackson (1991), Long-term earthquake clustering, Geophys. J. Int., 104, 117-133.

Kanamori, H., and D. L. Anderson (1975), Theoretical basis for some empirical realtions in seismology, Bull. Seism. Soc. Am., 65, 1073-1095. 
Keilis-Borok, V. (1959), On estimation of the displacement in an earthquake source and of source dimensions, Annali di Geofisica, 12, 2,205-2,214.

Kilb, D., J. Gomberg, and P. Bodin (2002), Aftershock triggering by complete coulomb stress changes, J. Geophys. Res., 10\%, doi:10.1029/2001JB0002002.

King, G., and M. Cocco (2001), Fault interaction by elastic stress. changes: New clues from earthquake sequences, Adv. Geophys., 44, 1-39.

Knopoff, L. (1957), Energy release in earthquake, Tech. Rep. 90, Institute of Gephysics, University of California, U.S.A.

Kostrov, B., and S. Das (1998), Principles of Earthquake Source Mechanics, Cambridge University Press.

Marsan, D. (2003), Triggering of seismicity at short timescales following Californian earthquakes, J. Geophys. Res., 108(B5), 2266, doi:10.1029/2002JB001946.

Mendoza, C., and S. Hartzell (1988), Aftershock patterns and main shock faulting, Bull. Seism. Soc. Am., 78, 1438-1449.

Nostro, C., L. Chiaraluce, M. Cocco, D. Baumont, and O. Scotti (2005), Coulomb stress changes caused by repeated normal faulting earthquakes during the 1997 Umbria-Marche (central Italy) seismic sequence, J. Geophys. Res., 110, B05S20, doi:10.1029/2004JB003386.

Ogata, Y. (1988), Statistical models for earthquake occurrence and residual analysis for point processes, J. Amer. Statist. Assoc., 83, 9-27.

Ogata, Y. (1998), Space-time point-process models for earthquake occurrences, Ann. Inst. Statist. Math., 50, 379-402.

Okada, Y. (1985), Surface deformation due to shear and tensile faults in an half-space, Bull. Seism. Soc. Am., $75(4), 1135-1154$.

Okada, Y. (1992), Internal deformation due to shear and tensile faults in an half-space, Bull. Seism. Soc. Am., 82(2), 10181040. 
Ruina, A. (1983), Slip instability and state variable friction laws, J. Geophys. Res., 88(B12), $10,359-10,370$.

Stein, R. (1999), The role of stress transfer in earthquake occurrence, Nature, 402, 605-609.

Stein, R., A. Barka, and J. Dieterich (1997), Progressive failure on the North Anatolian fault since 1939 by earthquake stress triggering, Geophy. J. Int., 128, 594-604.

Toda, S., and R. Stein (2000), Did stress triggering cause the large off-fault aftershocks of the 25 March 1998 Mw=8.1 Antarctic plate earthquake?, Geophys. Res. Lett., 27(15), 2301-2304.

Toda, S., and R. Stein (2002), Response of the San Andreas fault to the 1983 Coalinga-Nunez earthquakes: an application of interaction-based probabilities for Parkfield, J. Geophys. Res., 107 (B6), 2126, doi:10.1029/2001JB000172.

Toda, S., and R. Stein (2003), Toggling of seismicity by the 1997 Kagoshima earthquake couplet: a demonstration of time-dependent stress transfer, J. Geophys. Res., 108(B12), 2567, doi: 10.1029/2003JB002527.

Toda, S., R. Stein, P. Reasenberg, J. Dieterich, and A. Yoshida (1998), Stress transferred by the $1995 \mathrm{Mw}=6.9$ Kobe, Japan, shock: Effect on aftershocks and future earthquake probabilities, J. Geophys. Res., 103(B10), 24,54324,566.

Udias, A. (1999), Principles of Seismology, Cambridge University Press.

Utsu, T. (1969), Aftershocks and earthquake statistics (i) - investigation of aftershocks and other earthquake sequences based on a new classification of earthquake sequences, $J$. of Faculty of Science, Hokkaido Univ., Series VII (Geophysics), 3.

Utsu, T., Y. Ogata, and R. Matsuura (1995), The centenary of the Omori formula for a decay law of aftershock activity, J. Phys. Earth, 43, 1-33.

Wiemer, S. (2000), Introducing probabilistic aftershock hazard mapping, Geophys. Res. Lett., $27(20), 3405-3408$. 
Wiemer, S., and K. Katsumata (1999), Spatial variability of seismicity parameters in aftershock zones, J. Geophys. Res., 104(B6), 13,135-13,152.

Wiemer, S., and M. Wyss (2002), Spatial and temporal variability of the b-value in seismogenic volumes, Adv. Geophysics, 45, 259-302.

Wiemer, S., M. Gerstenberger, and E. Hauksson (2002), Properties of the Aftershock Sequence of the 1999 Mw 7.1 Hector Mine Earthquake: implications for aftershock hazard, Bull. Seism. Soc. Am., 92(4), 1127-1240.

Yamanaka, Y., and K. Shimazaki (1990), Scaling relationship between the number of aftershocksand the size of the mainshock, J. Phys. Earth., 38, 305-324.

Ziv, A. (2003), Foreshocks, aftershocks, and remote triggering in quasi-static fault models, $J$. of Geophys. Res. (Solid Earth), 108, 14,1-14,13.

Ziv, A., and A. M. Rubin (2003), Implications of rate-and-state friction for properties of aftershock sequence: Quasi-static inherently discrete simulations, J. of Geophys. Res., 108, 2051, doi:10.1029/2001JB001219. 


\section{Figure captions}

Figure (1): Conventions adopted for the reference system and for the parameters of the orientation of a fault: the strike $\phi, \operatorname{dip} \delta$ and rake $\lambda$ angles and the unit vectors $\mathbf{n}$ and $\mathbf{l}$. The $x_{3}$ axis is positive toward the Nadir direction.

Figure (2): Shear stress, $\sigma_{12}$, parallel to the slip direction for a strike-slip fault projected on the fault plane $\left(x_{1} x_{3}, x_{2}=0.2 \mathrm{~km}\right)$.

Figure (3): Distribution of the triggered seismicity against time for different values of induced shear stress. The values of the parameters used are: $r=2$ events $/ y, \Delta \tau=3 M P a$, $A \sigma=0.24$ and $\dot{\tau}=5 \mathrm{KPa} / \mathrm{y}$.

Figure (4): Distribution of the number of expected events in four different cases: (a) for a positive value of earthquake shear stress equal to $5 \mathrm{MPa}$, (b) for a negative value of it equal to $-5 \mathrm{MPa},(\mathrm{c})$ the sum of (a) and (b) and (d) the integral of (c) over time. The values of $r$ and $t_{a}$ are fixed at 1 .

Figure (5): Representation of the total number of induced events (on vertical axis) in six different arbitrary slices (red, green and blue columns) around the principal fault after different intervals of time (on horizontal axis). The distances of the limits of the slices are computed from the perimeter of the source and the reference magnitude in the simulation is 6.0. Histogram (a) represents the distribution nearest to the source at shorter intervals of time (the time scale is purely qualitative). Histogram (b) represents the distribution farther from the fault at longer intervals of time. 
Figure (6): Spatial distribution of the density of induced events at $t=0.01$ year.

Figure (7): Spatial distribution of the density of induced events at $t=1$ year.

Figure (8): Spatial distribution of the density of induced events at $t=100$ year.

Figure (9): Comparison between the temporal distribution of the cumulative number of induced events corresponding to synthetic data and the best fits obtained by the Omori law for the closer slices from the source.

Figure (10): Comparison between the temporal distribution of the cumulative number of induced events corresponding to synthetic data and the best fits obtained by the Omori law for the farther slices from the source. 


\section{Figures}

Figure 1:

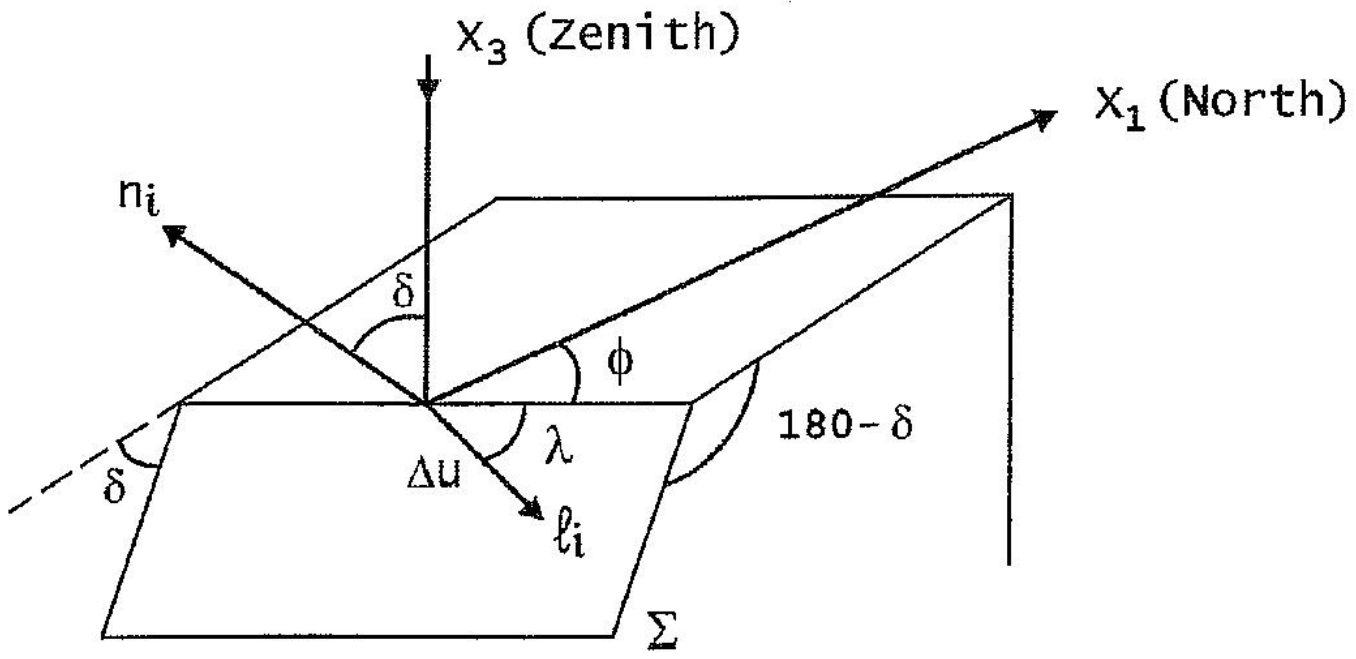

Figure 2:




Figure 3:

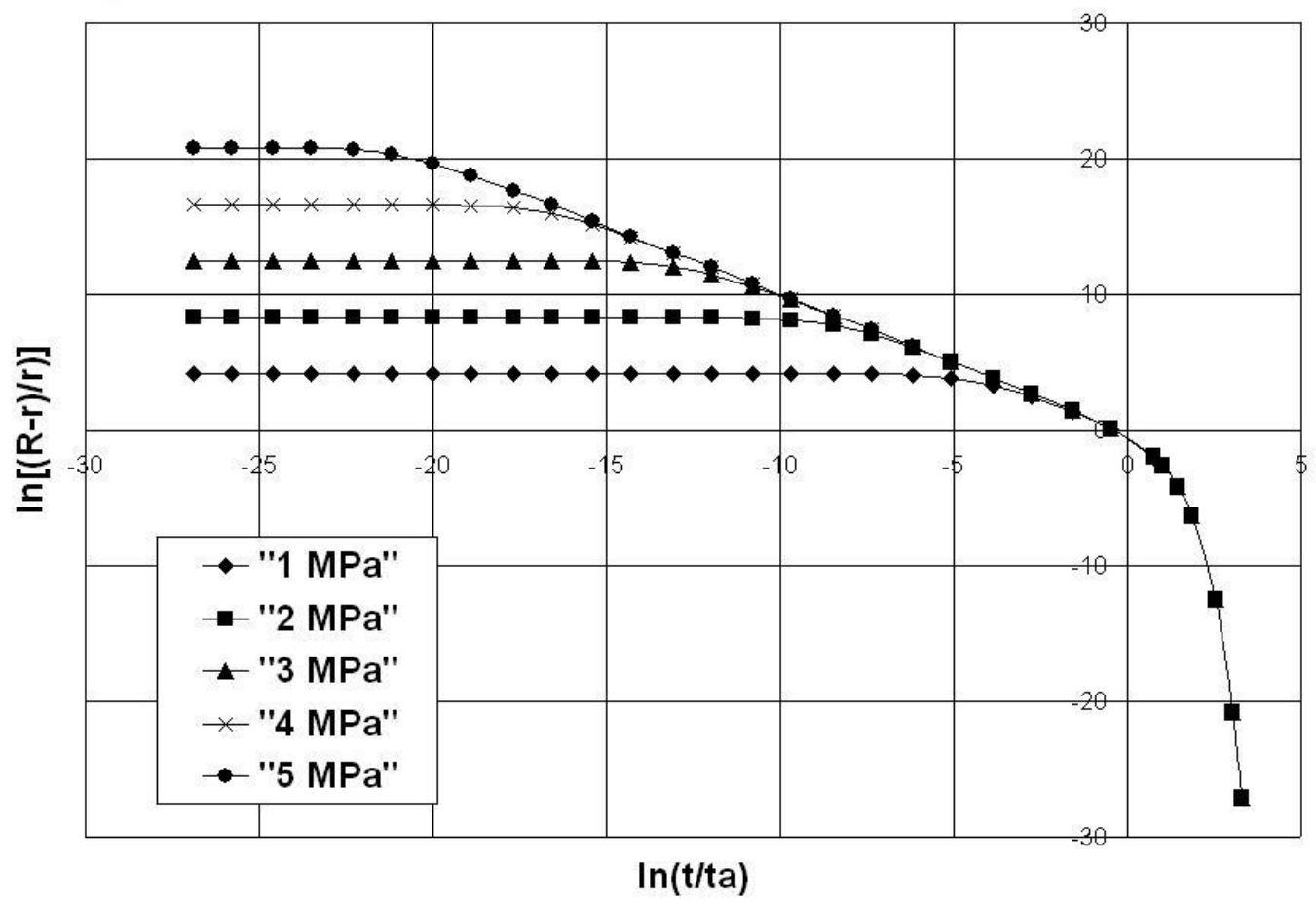

Figure 4:

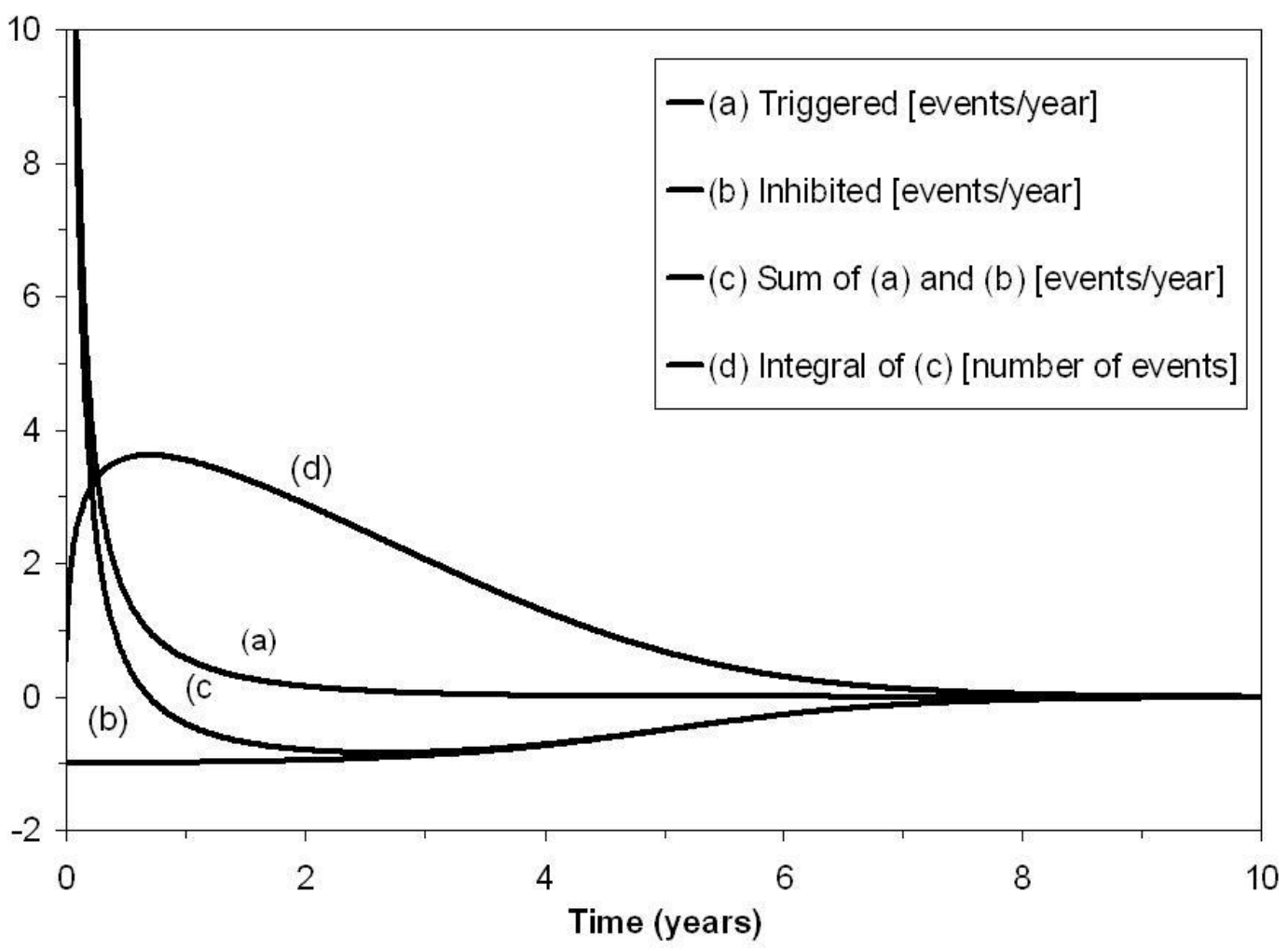


Figure 5:
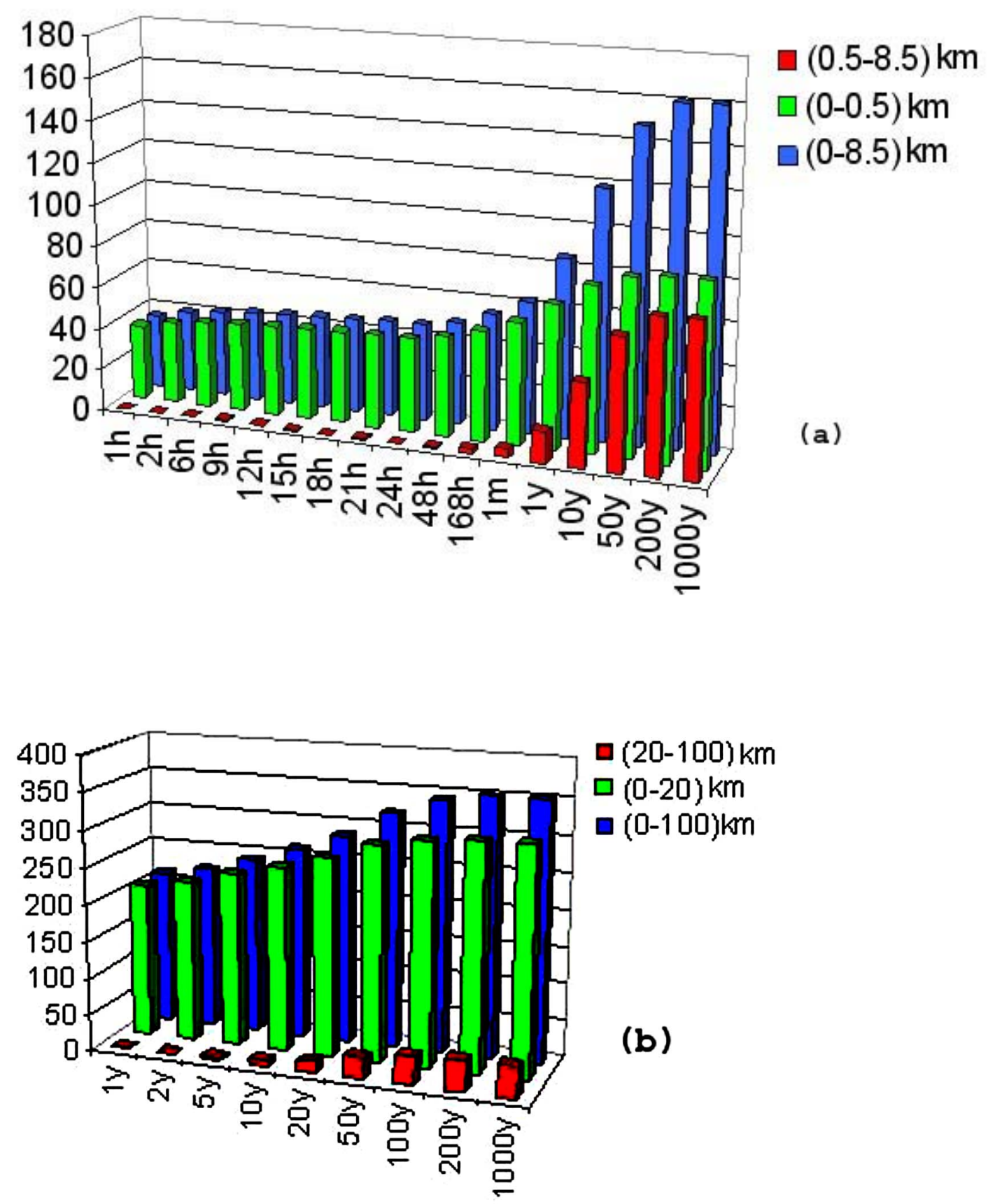
Figure 6:

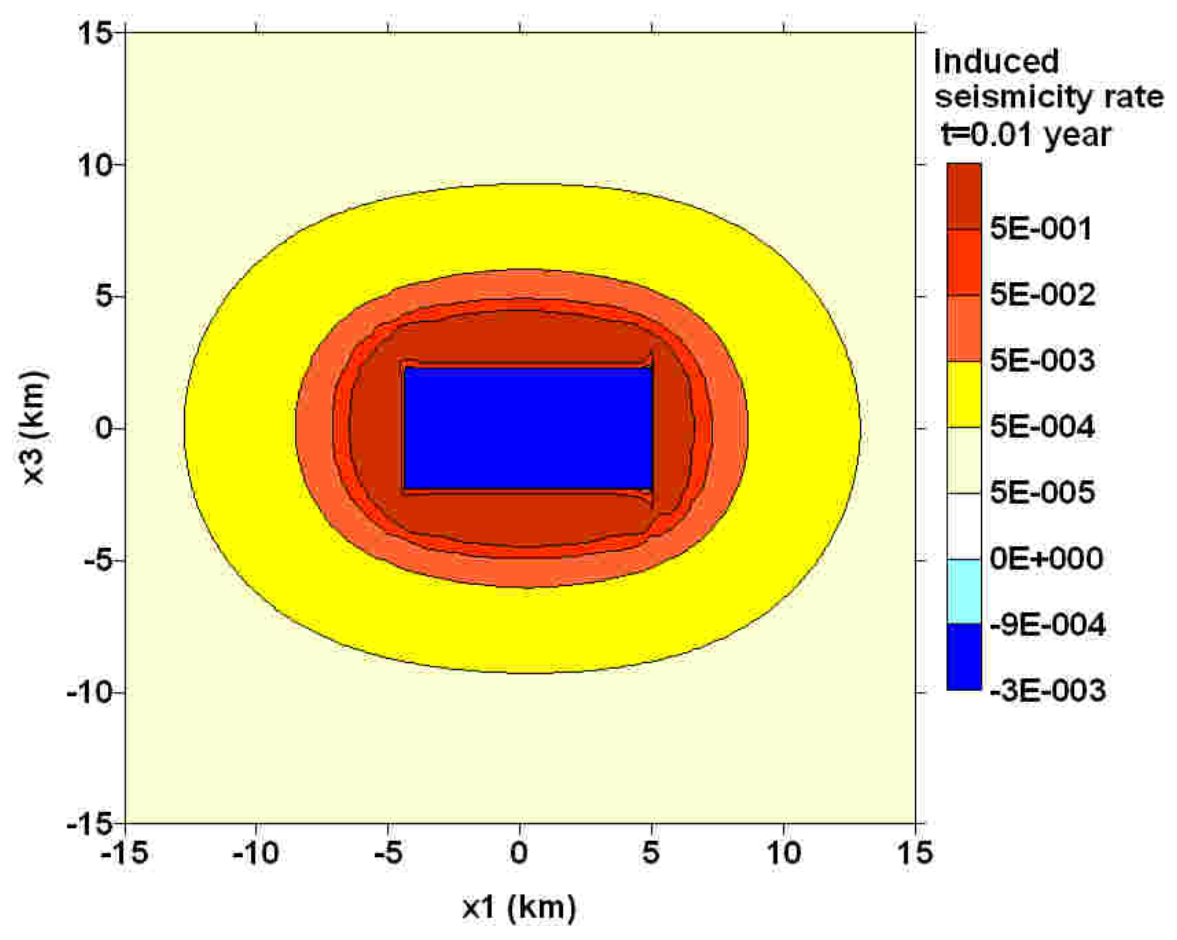

Figure 7:

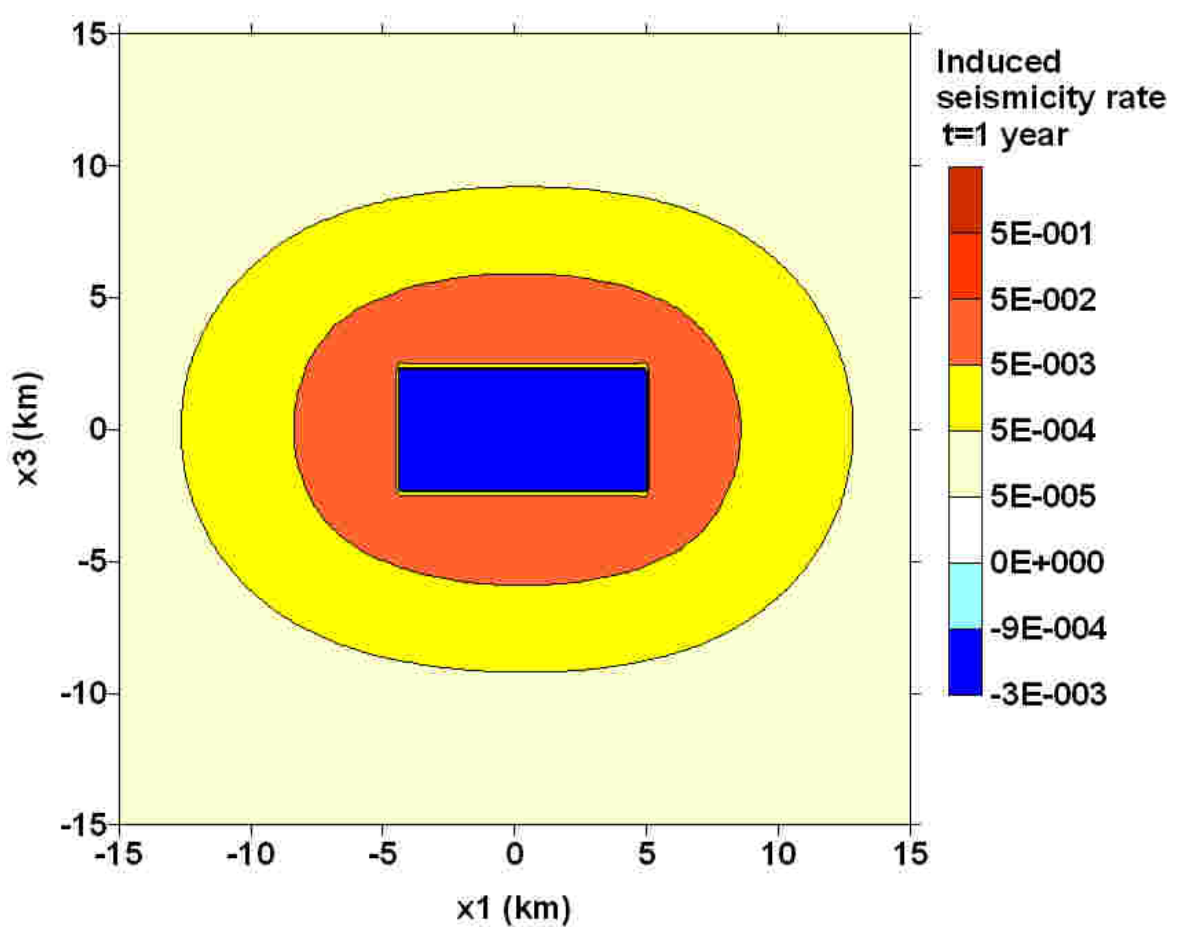


Figure 8:

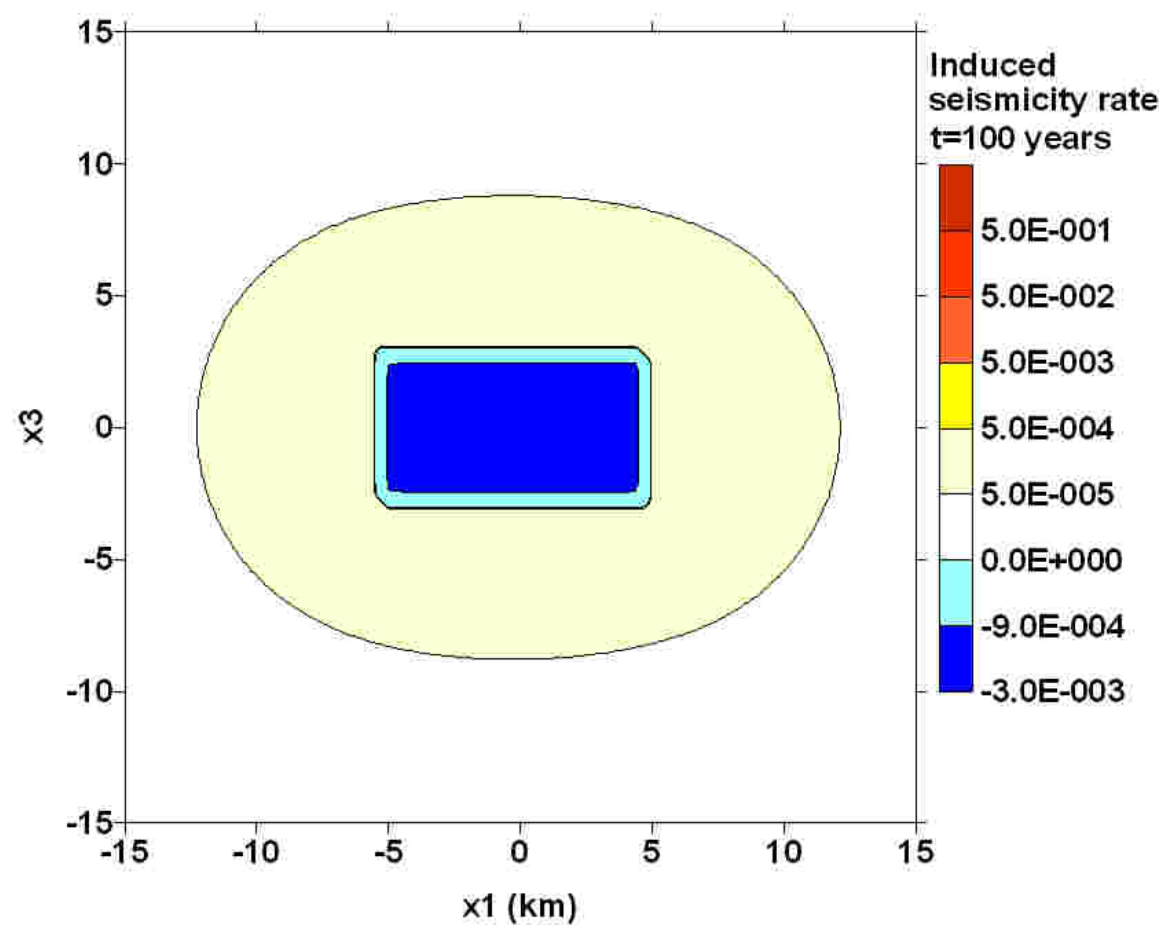


Figure 9:

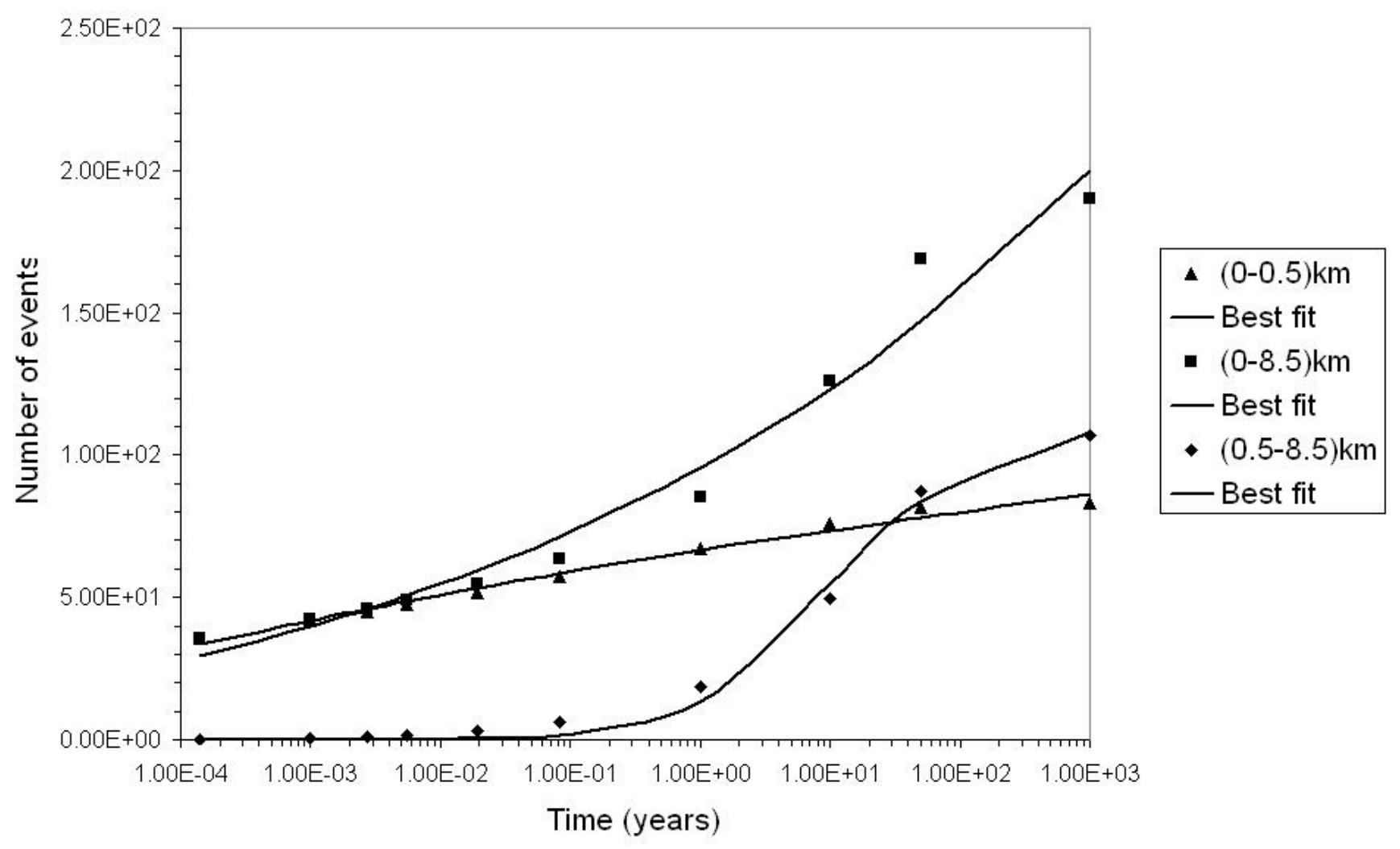

Figure 10:

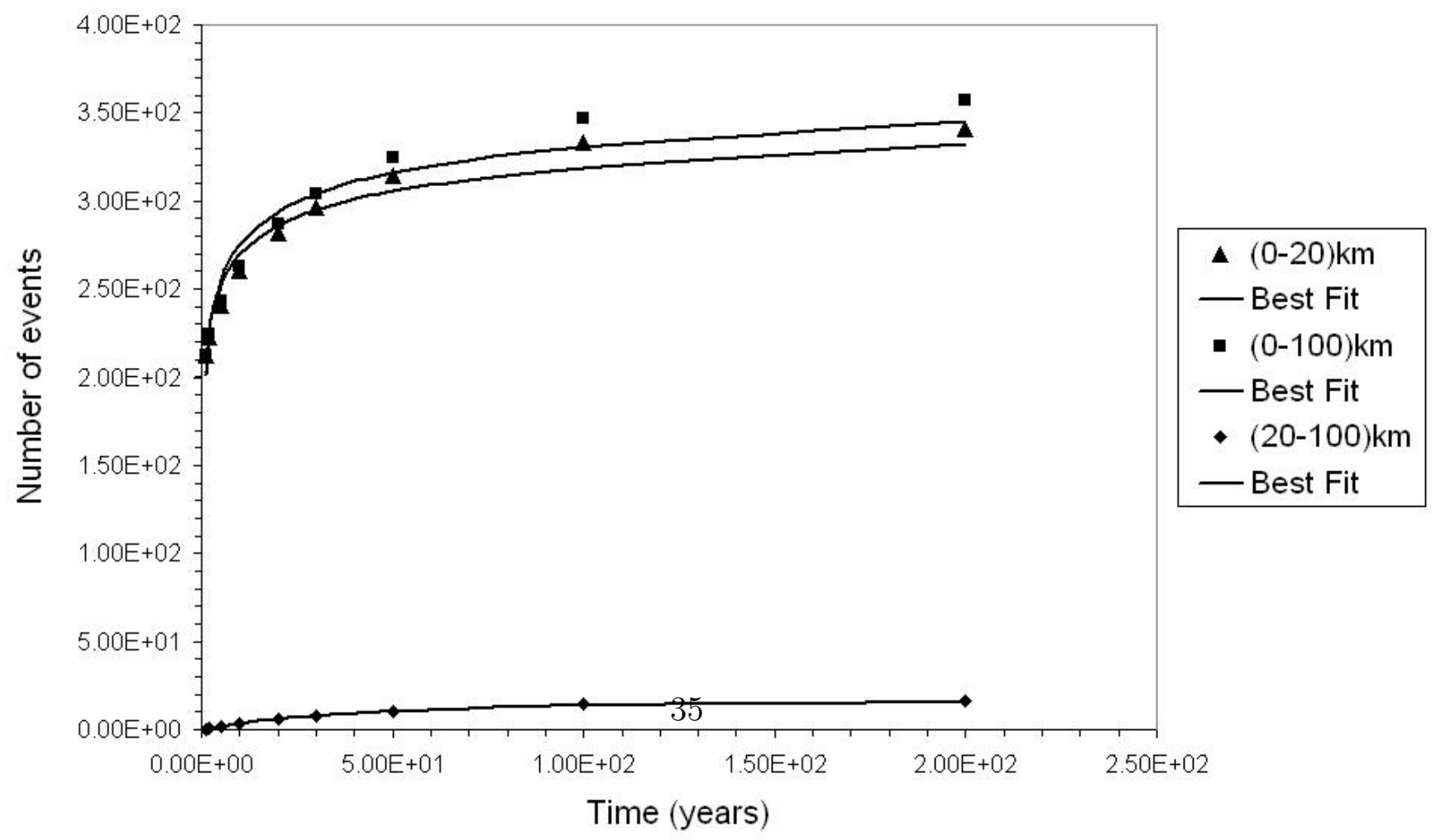

